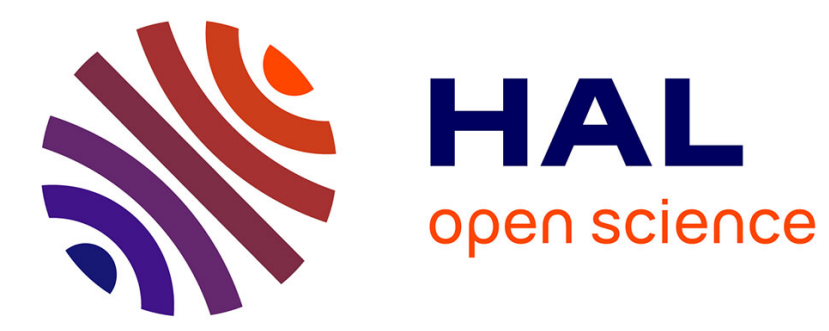

\title{
Principal Concepts of Martensitic Theory
}

A. Roytburd

\section{To cite this version:}

A. Roytburd. Principal Concepts of Martensitic Theory. Journal de Physique IV Proceedings, 1995, 05 (C8), pp.C8-21-C8-30. 10.1051/jp4:1995803 . jpa-00254051

\section{HAL Id: jpa-00254051 https://hal.science/jpa-00254051}

Submitted on 1 Jan 1995

HAL is a multi-disciplinary open access archive for the deposit and dissemination of scientific research documents, whether they are published or not. The documents may come from teaching and research institutions in France or abroad, or from public or private research centers.
L'archive ouverte pluridisciplinaire HAL, est destinée au dépôt et à la diffusion de documents scientifiques de niveau recherche, publiés ou non, émanant des établissements d'enseignement et de recherche français ou étrangers, des laboratoires publics ou privés. 


\title{
Principal Concepts of Martensitic Theory
}

\author{
A.L. Roytburd \\ Department of Materials and Nuclear Engineering, University of Maryland at College Park, College Park \\ MD 20742, U.S.A.
}

\begin{abstract}
Fundamentals of the theory of martensitic transformations are discussed. Principal theoretical concepts which are valid for all phase transformations in solids are formulated and illustrated. Some basic problems which are still unsolved are pointed out.
\end{abstract}

\section{INTRODUCTION}

This paper is devoted to the discussion of some principal concepts of the theory of martensitic transformations:

\section{a. Phase-field approach to the description of the evolution of heterophase states}

Considering a martensitic transformation as the time evolution of a strain field has opened the way to the understanding and quantitative description of nucleation and growth of martensite phases. The theory of the soliton-like movement of interfaces allows one to consider the dynamics of martensitic transformations. The ideas of non-classical nucleation enable one to understand the kinetics of martensitic transformations close to unstable regions. The phase-field approach establishes a common background for first-order (martensitic) and second-order (ferroelastic) transformations. However, for strong first-order martensitic transformations, the classical approach based on the macroscopic concept of sharp interfaces still remains adequate.

\section{b. Polydomain phases in the thermodynamics of coherent phases}

Coherency and internal stresses are the factors that determine the thermodynamics and polydomain microstructures of martensitic transformations. Polydomain phases can be made up of an equilibrium mixture of domains of parent and product phases, including phases which exist only under stress. The most important feature of polydomain phases is that they can reversibly change their domain structure resulting in the shape memory effect and superelasticity.

\section{c. Interaction and competition between martensitic transformation and plastic} transformation

Martensitic transformation in real materials is closely knit with plastic deformation which gives a universal mechanism for the relaxation of nternal stress. The interaction of nucleating and moving martensite interfaces with dislocations controls the real kinetics of martensitic transformations. The analysis of the effects of dislocations on the nucleation and the growth of martensite allows one to describe a broad spectrum of martensitic and martensitic-like transformations from the athermal to self-diffusional kinetics.

These concepts were developed on the basis of experimental and theoretical studies of martensitic transformations to provide an explanation to this important phenomena. However, they have also been found satisfactory and effective towards the understanding of all phase transformations including decomposition and ordering (atomic, ferroelectric and ferromagnetic).

\section{MARTENSITIC TRANSFORMATION AS A STRAIN PHASE TRANSFORMATION}

A phase transformation in solids is associated with the change of one or several parameters determining the phase state. There can be three types of transformation parameters: composition, order parameters (e.g., atomic ordering, ferroelectric or ferromagnetic ordering) and parameters which describe the mutual arrangement of crystal lattice sites. The displacement of one sublattice with respect to another may be an example of lattice transformation (Fig. 1). The change of transformation parameters as a rule are accompanied by some strain of a crystal lattice or by self-strain. Meanwhile, there are transformations for which the strain itself is the only transformation parameter. Martensitic transformations are considered as strain phase transformations in which states of phases are completely determined by the strain of the lattice 
$[1,2]$. A strain phase transformation in a more general case can include, in addition to the homogeneous strain, some kind of rearrangement or shuffling of lattice points, provided that this shuffling is mutually and unambiguously related to the strain. Apparently, such transformations as f.c.c. $\Leftrightarrow$ h.c.p. and b.c.c. $\Leftrightarrow$ h.c.p., which are associated with pairwise sliding of close-packed planes, and are related to strain transformations.

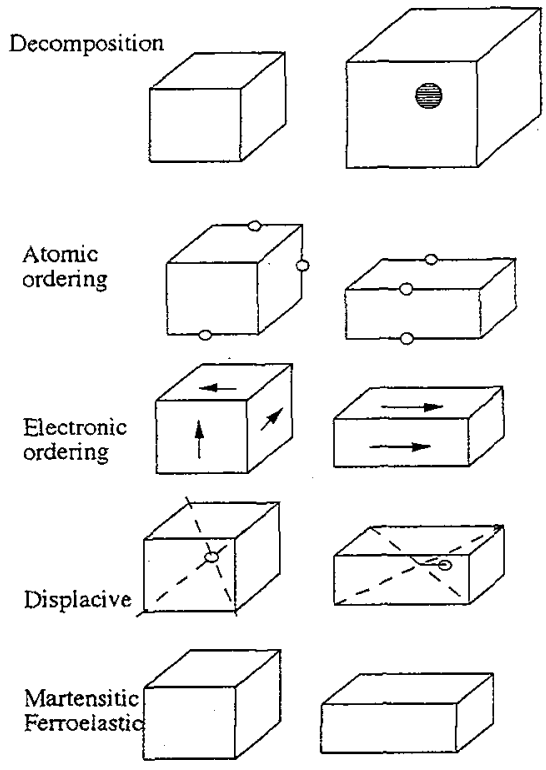

Figure 1: Self-strain of different types of solid-solid phase transformations.

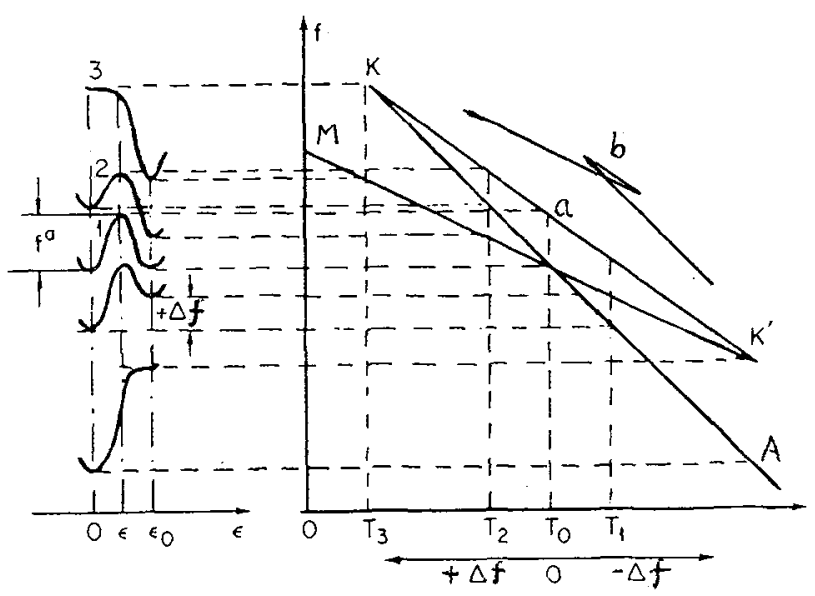

Figure 2: Free energy density for a strain phase transformation.

The free energy density $f$ of the homogeneous phases as a function of the transformation parameters in a strain phase transformation can be represented as a surface in the space of independent components of strain tensor $\hat{\varepsilon}$. The equilibrium states of the phases correspond to the minima in this surface. In particular, the curvature $\partial^{2} f / \partial \hat{\varepsilon}^{2}$ in the vicinity of the minima determines the elasticity modulus $\hat{G}$. The minima are separated by a "ridge", the saddle point of which corresponds to the saddle state during the transformation and is characterized by the activation energy $f^{a}$.

Figure 2 demonstrates schematically the positions of the minima and saddle points of the free energy $f(\hat{\varepsilon})$ as a function of temperature. Under isothermal conditions, the system passes from the point of the metastable trough in the surface $f(\hat{\varepsilon})$ across the barrier to the more stable trough. The temperature $T_{o}$ at which the energies of the initial and the final states are equal is the phase equilibrium point for the first order transformation. In principle, the lines of the metastable equilibrium states end up at the points where some tensor components of curvature $\partial^{2} f / \partial \hat{\varepsilon}^{2}$ vanish and the trough degenerates: the homogeneous phase loses its stability (points $K$ and $K^{\prime}$ in Fig. 2).

The activation energy may be estimated as follows:

$$
f^{\mathrm{a}}=\frac{1}{4} e_{\mathrm{o}}=\frac{3}{2} G \varepsilon_{\mathrm{o}}^{2},
$$


where $e_{\mathrm{o}}=(1 / 2) G \varepsilon_{\mathrm{o}}{ }^{2}$ is the characteristic energy and $\varepsilon_{\mathrm{o}}$ is the self-strain. By virtue of the smallness of the self-strain, $f^{a}$ is small compared with the interatomic binding energy, which per unit volume is of the same order of magnitude as $G$.

In order to assess the possibility of loss of stability of the metastable homogeneous phase, it is necessary to compare $f^{\mathrm{a}}$ with the attainable value of the driving force $\Delta f$. For transformations at cooling, the maximum $\Delta f$ value at $0 \mathrm{~K}$ is of the same order of magnitude as the latent heat $q$ the of transformation. If $f a / q$ is small, the temperature interval of metastability of the parent phase is narrow $\left[\left(\mathrm{T}_{\mathrm{o}}-\mathrm{T}_{\mathrm{K}}\right) / \mathrm{T}_{\mathrm{o}} \approx f^{\mathrm{a}} / q\right]$, and the elastic modulus decreases with the deviation from equilibrium temperature $T_{o}$ and ends up to be zero at $\mathrm{T}_{\mathrm{K}}$. This is a weak first-order phase transformation, close to a second order one (curve $\mathrm{b}$ in Fig. 2). If $f^{\mathrm{a}} / q>1$ there is no loss of stability of the parent phase. For the martensitic transformation in Fe-based alloys, $f^{\mathrm{a}} / q \approx 10$ and there is no evidence of considerable dependence of the elastic modulus on the temperature. This is a strong first-order martensitic transformation.

There are two facts that are worth noting in connection with the strain nature of the martensitic transformation:

1. External stress has a strong effect on the transformation. Since $\hat{\sigma}=\partial f / \partial \hat{\varepsilon}$, the thermodynamics of the transformation under stress is determined by the Gibbs free energy $g=(f-\hat{\sigma} \hat{\varepsilon})$. The phase equilibrium corresponds to $\Delta g=\Delta f-\hat{\sigma} \hat{\varepsilon}_{0}=0$. This equation expresses the Clausius-Clapeyron relation for the shift of the phase equilibrium under external stress.

2. The stress changes the interval of existence of the metastable phases. Therefore, the stress can stabilize the phases which do not exist at all without the stress.

The knowledge of the free energy is necessary step to a quantitative martensitic theory. The first microscopic calculations yield important information and provided hope that this approach will be completed in the near future [3].

\section{COHERENCY: CONTINUITY OF STRAIN OR CONTINUITY OF DISPLACEMENT?}

As in all first order transformations, the martensitic transformation also proceeds through the evolution of a heterophase state consisting of a parent ("austenite") and a product ("martensite") phases. For a strain phase transition, if the self-strain is not very large $\left(\varepsilon_{0}<1\right)$, it is natural to assume that during the transformation in a crystal, the continuity of the crystal lattice is not violated or that the crystal lattice remains coherent even in a heterophase state. The concept of coherency was formulated as a result of experimental studies of the martensitic transformation [4] and is fundamental to the theory of phase transformations in solids.

Strain phase transformation in a coherent crystal can be considered as the evolution of a strain field. To describe the evolution, it is necessary to connect a change in the strain with time at each point of the crystal with the corresponding change in the free energy of the crystal. The evolution equation should include time derivatives of a local strain $(\dot{\varepsilon}(\mathrm{r}, \mathrm{t}), \ddot{\varepsilon}(\mathrm{r}, \mathrm{t})$, etc.) and a variational derivative of the free energy $\delta \mathrm{F} / \delta \varepsilon$. The free energy of the crystal may be expressed as:

$$
F=\int_{V} f(\hat{\varepsilon}, \nabla \hat{\varepsilon}) \mathrm{d} V \cong \int_{V}\left[f(\hat{\varepsilon})+f^{\mathrm{a}} l^{2}(\nabla \hat{\boldsymbol{\varepsilon}})^{2}\right] \mathrm{d} V
$$

where the local free energy depends not only on the strain $\hat{\varepsilon}(r)$ itself but also on the strain gradient, $\nabla \hat{\varepsilon}$. If the gradient is not very large, the dependence on the gradient may be approximated by the quadratic term only. The coefficient of this term can be expressed through the characteristic energy $f^{\mathrm{a}}$ and a characteristic length $l$. The length $l$ represents the thickness of interfaces in the heterophase crystal which are intermediate layers between phases (Fig. 3). The gradient term in Eq. (2) is responsible for the short-range interaction between the points of a transforming crystal. There is also long-term, elastic interaction because the transformation parameter $\hat{\varepsilon}$ is the gradient of displacement $\mathbf{u}$; and the free energy does not depend on displacement itself.

The field approach to martensitic transformation was first proposed in [5,6]. It was shown in these papers that the movement of the austenite-martensite interface is described by the same dynamic equations as a Frenkel-Kontorova dislocation. The Peierls' barrier for the interface movement (the amplitude of the periodic change of the energy during the movement of the interface through a periodic potential of a crystal lattice) was shown to be small as was the surface energy of the interface. The field approach can be 
generalized to include nucleation events and the energy of nucleation was estimated for some field models $[1,7]$.

The field theory of soliton-like movement of interphase and intertwin interfaces has since been developed further and the parameters of the theory has been related to dynamic properties of crystals $[8,9]$. The effects of heat diffusion, isothermal and adiabatic conditions of interface movement were analyzed in [10]. The nucleation aspects of the field theory were reflected in analysis of non-classical nucleation $[3,11,12]$.
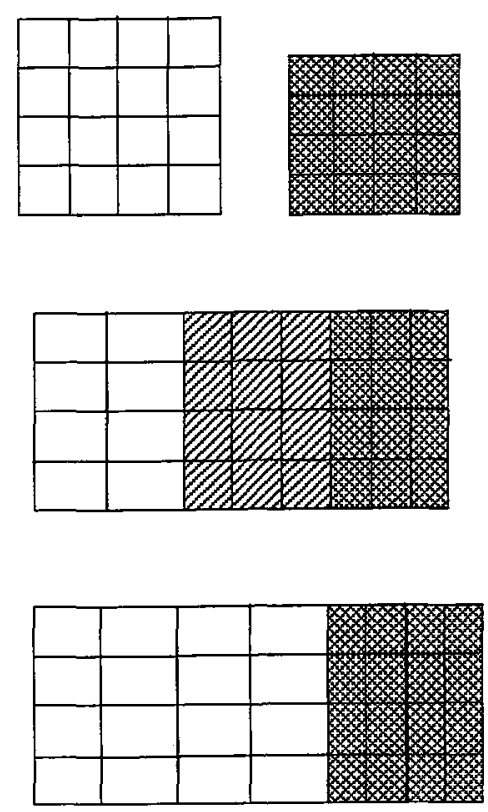

Self-Strain + Coherency<smiles>[Te][Te]</smiles>

Internal Stress and Elastic Energy

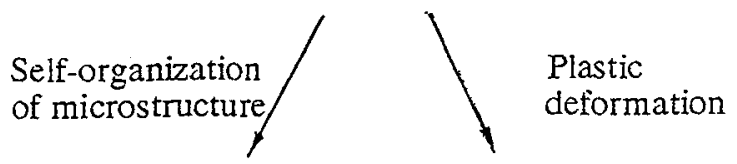

Stress Relief and Relaxation of Energy

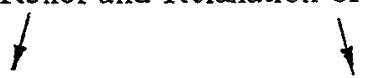

Equilibrium

arrangement of

polydomain

plates

Dislocation

screening and

formation of

incoherent

interfaces

Figure 3: Smooth and sharp coherent interfaces.

Figure 4: Two ways of relaxation of martensitic structures.

The works on the field theory of martensitic transformation are in the mainstream of modern theory of phase transformations. Due to its mathematical self-consistency and computational convenience, the phase field approach is widely applied to the analysis of diffusional transformations, crystallization, and ferromagnetic transformations. However, for martensitic transformations its application is likely to be restricted by weak transformation near critical points ("quasimartensite", [3]). The restriction is connected with the common approximation of the of the free energy dependence on the gradient (Eq(2)), which is valid only if the characteristic length is large in comparison with interatomic distance. It is correct for diffusional transformation or for transformation near the limit of stability (critical points), but it is not correct for strong first order transformations. For these kind of transformations, the interface thickness is close to the interatomic distance, and the concept of a sharp interface is a more appropriate description of reality. In the case of a sharp interface coherency implies continuity of the displacement of lattice sites. The strain component parallel to the interface is continuous while the normal components can be discontinuous. This condition of strain compatibility makes it possible to determine the elastic field created by an interface independent of the exact atomic structure of the interface. The interface structure determines the surface energy of an interface which is (besides the parameters describing the thermodynamic properties of the elastically distorted phases) a fundamental parameter of the macroscopic theory of martensitic transformations [12]. 
The kinetic condition of the transformation not very far from equilibrium is described by a local equation for all interface points as follows:

$$
v=\mathrm{k} \Delta g ; \quad \Delta g=(\Delta f-\hat{\sigma} \Delta \hat{\varepsilon})
$$

where $v$ is the interface velocity, $\mathrm{k}$ is a mobility coefficient, $\Delta \mathrm{g}=\Delta f-\hat{\sigma} \Delta \hat{\varepsilon}$ is a local driving force with $\Delta f$ and $\Delta \hat{\varepsilon}$ are the differences between the free energies and the strain on both sides of the interface and $\hat{\sigma}$ is a local stress at the interface [1]. For conditions which are far from equilibrium the movement of the interface is more similar to a shock wave than a soliton $[13,14]$.

The energy and the Peierls' barrier for a sharp interface are relatively small, $\Gamma \cong \Delta \Gamma \cong f^{a} \mathrm{~b}$. The barrier disappears at small driving force and the interface movement becomes independent of thermal activation [2]. On the other hand, coherent interfaces are a source of internal stress. Their elastic energy is minimum if the interface is planar and is specially oriented [1]. It results in relative rigidity of the interface so it cannot be pinned by microscopic obstacles: point defects or individual dislocations.

\section{SELF-ORGANIZATION OF MARTENSITIC TRANSFORMATION; POLYDOMAIN MICROSTRUCTURES}

The incompatibility of the transformation self-strain in a coherent crystal results in internal stresses and elastic energy. However, there are relaxation mechanisms which prevent accumulation of considerable elastic energy. These are self-organization of the microstructure and plastic deformation (Fig. 4). The interaction and the competition between these mechanisms of relaxation determine many observed features of martensitic transformations.

The self-organization leads to the formation of equilibrium microstructures which correspond to the minima of the heterophase crystal free energy. The requirement that the configuration driving force $\Delta g$ (Eq.(3)) be equal to zero at all non-fixed points of the interfaces gives the equation for determining the equilibrium microstructures [1]. The solutions of these equation describe regular essembles of specially oriented martensite plates. The martensite plates usually have polydomain structures, i.e., they consist of sets of alternating domains. The domains correspond to different crystallographic variants of a transformation because the martensite phase has a lower symmetry than the parent austenite. The possible polydomain structures generated by cubic-tetragonal transformations (e.g., b.c.c $\Leftrightarrow$ f.c.c.) are shown in Figure 5. The simplest structures (polytwins) contain pairs of domains. The second-order structure contains 2 pairs of poytwins. Hierarchical polydomain structures have more degrees of freedom to adjust the transformation self-strain and therefore to minimize the elastic energy further. Examples of equilibrium martensite plates embedded in an austenite matrix are shown in Figure 6. The polytwin plates minimize the elastic energy concentrated inside the plates; the plate with a second order domain structure additionally minimizes the elastic energy of the stress field near the plate edge.

The equilibrium heterophase microstructure should change under external stress [15]. If the interfaces are mobile, it will result in some easy modes of the deformation, which can be reversible ("superelastic deformation", Fig. 7). For microstructures containing austenite the $\sigma-\varepsilon$ superelastic curves can demonstrate a negative elastic modulus or a thermodynamic hysteresis $[18,19]$.

Because of the considerable shear component of the self-strain, the equilibrium martensitic microstructures usually have almost no internal stress and therefore, correspond to zero elastic energy. The reason is that domains in a polytwin have compatible self-strains and average self-strain of a polytwin can be made compatible with other polytwins or austenite. Such kind of a stress-free microstructure is described by the relations of compatibility at the interfaces, which allows to find equilibrium microstructures of different orders of complexity. For example, the domain microstructures for cubicorthorhombic transformation is considered in detail in [20]. The equations of compatibility are purely geometrical and can be solved for any self-strain without the common approximation of small strain used by the theory of elasticity. This geometrically non-linear problem was solved by the invariant plane theory which successfully analyses the microstructural features of individual martensite plates $[21,22]$. The geometrically non-linear approach has been recently generalized to describe high-order martensitic structures. In particular, the systematic analysis of the possible compatible martensite microstructures has used to determine the strain, which can be obtained without violating the stress-free condition[23-25].

The capability of the elastic domain theory in the analysis of stress states and the accuracy of the geometrically non-linear theory can be combined if an exact solution of the non-linear theory is chosen as a reference state for the thermodynamical calculation [26]. This kind of an approach was used for the calculation of the effect of stress on the habitus and the microstructure of martensite plates in Fe-based alloys and a good agreement with the experimental observations was obtained [27]. 


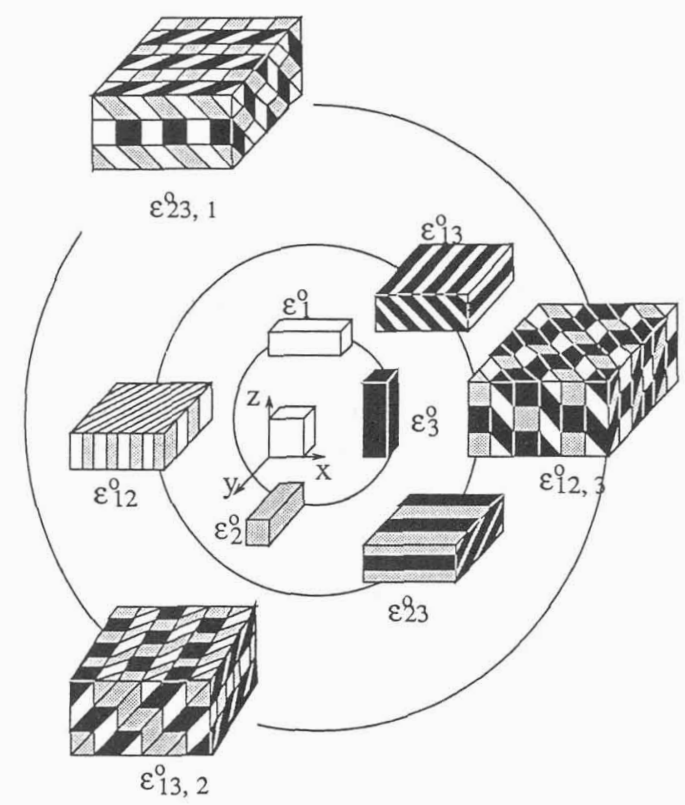

Figure 5: Polydomain microstructures for cubic-tetragonal transformation [16].
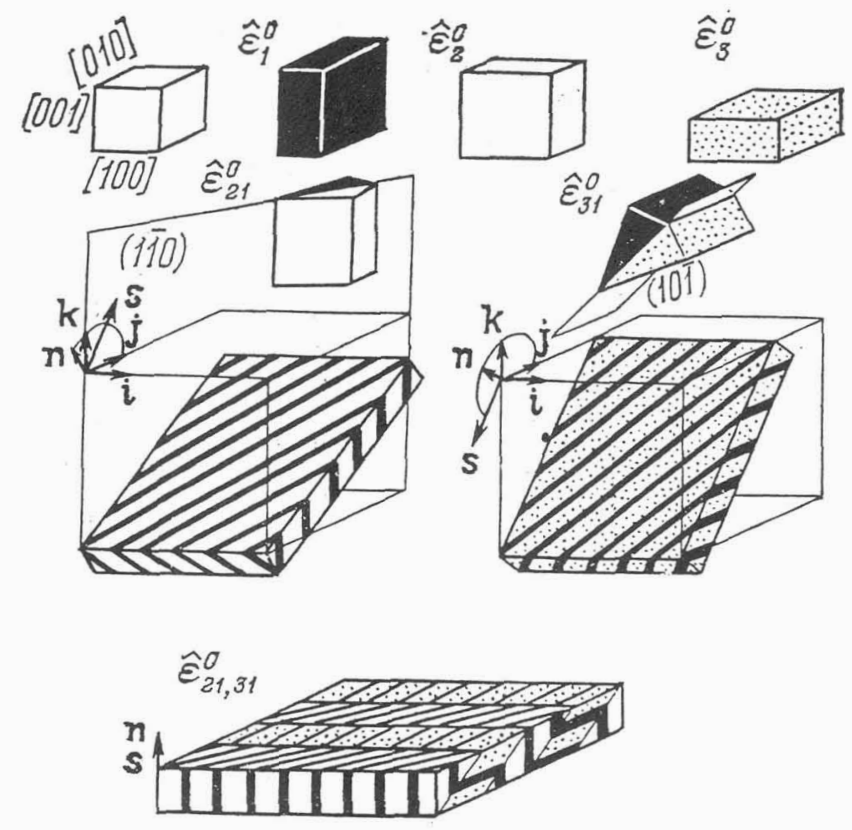

Figure 6: Equilibrium polydomain plates for cubic-tetragonal transformation [17]. 
Martensitic domains minimize the elastic energy just as other domains (e.g. magnetic) minimize the energy of other long-range fields and thus they are elastic domains. The concept of elastic domains is quite general and can be applied to all coherent phase transformations $[1,15,17,28]$. Non- $180^{\circ}$ domains in ferromagnets and ferroelastics are also elastic domains because of the striction strain. This implies that the domain structures of these materials can be governed by the combined effect of magnetic (electric) and mechanical external fields [16].

The important unsolved problems in the theory of martensitic microstructures are connected with the effects of inhomogeneities. Two limiting cases are evident: the effect of an inhomogeneous external field on a homogeneous microstructure and the effect of a homogeneous field on an inhomogeneous microstructure. The first was explored [29] and it was shown that martensitic microstructure should demonstrate unusual "gradient" elasticity, resulting in a strong resistance to non-uniform stress (e.g., at bending) together with a large compliance to uniform stress (e.g., elongation). This non-local effect should be taken into account in the analysis of the mechanics of martensitic materials. The behavior of macroscopically non-uniform polydomain microstructures (a single crystal with non-self-consistent polydomain fragments, polycrystals, composites, etc.) determines the shape memory and superelasticity of real materials. The first step in the theory of superelasticity of polycrystals has been done in [30].

The theory of polydomain microstructure is a macroscopic one (inspite of the word "microstructure"). This means that the effect of the interface energy is assumed to be a secondary one. However, there have been successful attempts to consider a polytwin structure on a microscopic scale where the contribution of interfaces in the energy of a system is comparable to the elastic and chemical volume energies. These include the nucleation of a polydomain phase [12] and the concept of microscopically adaptive phases [31]. I believe that atomistic calculations of the twin interface energy in alloys will give a solid basis for these ideas.

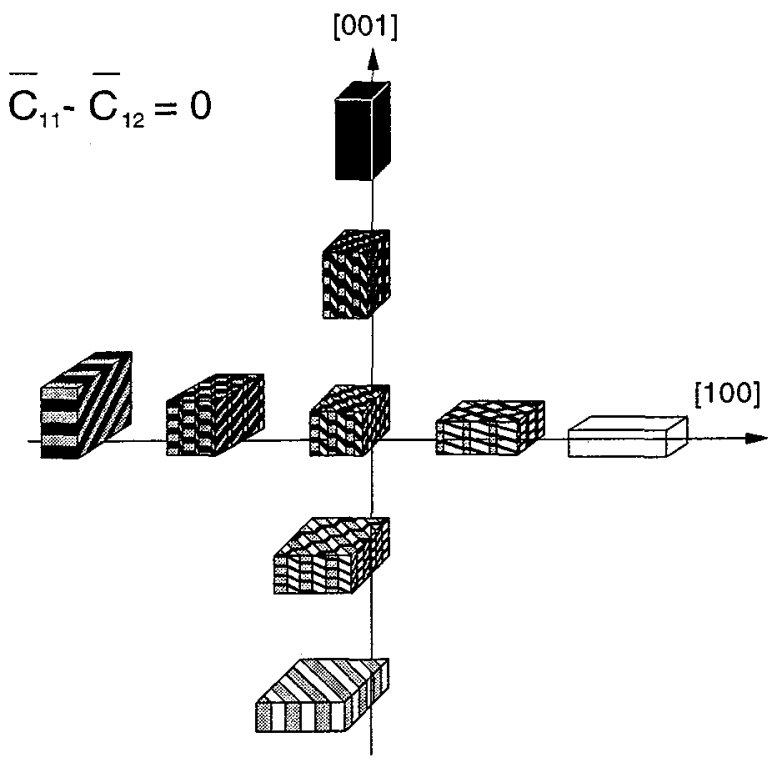

Figure 7: The easy (superelastic) modes of deformation of 3-domain microstructure. 


\section{EFFECTS OF INTERNAL STRESS RELAXATION DUE TO PLASTIC DEFORMATION}

Internal stresses in real crystals stimulate formation and movement of defects, especially dislocations. In fact, internal stresses cause the process of plastic deformation which can determine the thermodynamics and the kinetics of the transformation.

Nets of dislocations emerge on interfaces as a result of plastic deformation occurring in a new phase during its formation. The elastic fields of these interface dislocations compensate the fields of coherent segments of the interface. Therefore, partial conversion of the elastic energy into interfacial energy takes place. This effect can dramatically change the energy barrier for nucleation. On the other hand, the interface dislocations to a great extent define the mobility of interphase interfaces (Figure 8). Thus, the whole spectrum of kinetic types of the transformation can exist depending on degree of the plastic relaxation $[1,2]$.

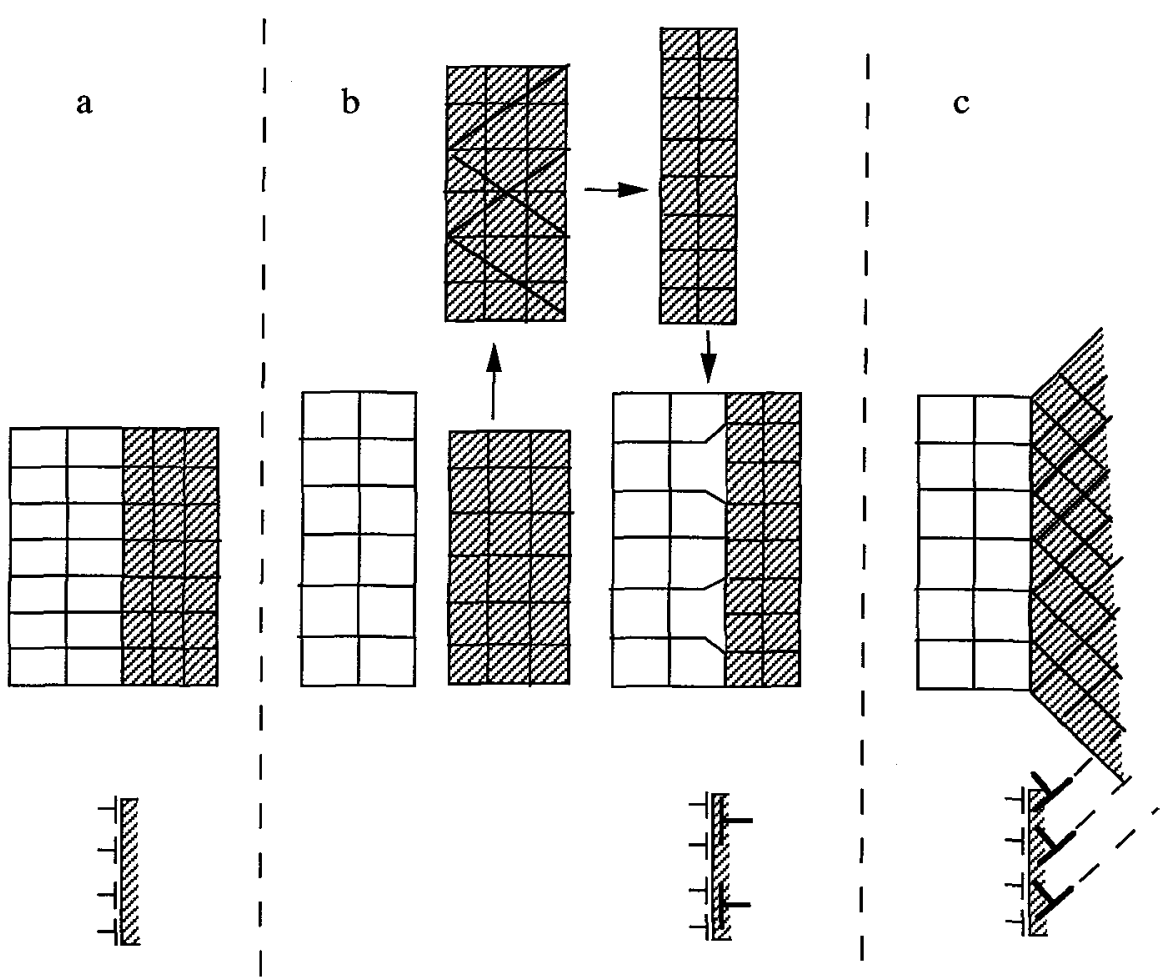

Figure 8: Formation of interface dislocations as a result of plastic deformation in martensite.

a. Coherent state.

b. Double slip results in the formation of climbing interface dislocations; the dislocation interface can move only due to the diffusion of vacancies or interstitial atoms.

c. Glissile dislocation interface.

The dislocation models of interfaces are shown at the bottom of the figure. 
The dislocation structure of the interface is in continuous development. Exchange of dislocations between the interface and the adjacent parts of the crystal takes place when the interface moves. The interface absorbs and emits dislocations. There are reactions of transformation of lattice dislocations into interface dislocations and back. Moreover, defects created in the process of the plastic deformation under external forces can significantly influence the development of the transformation. Certain defects and defects clusters can slow down the interface and serve as nucleation sites of the new phase. Therefore, the evolution of a heterophase system during the phase transformation is, in fact, the interconnected development of two subsystems. The first subsystem is the nucleating and growing of areas of the new phase. The other subsystem is the nucleating and moving lattice defects. The impact of the plastic deformation can be diminished only in particular cases. These cases include transformations at low temperature and transformations of perfect crystals. In these cases it becomes possible to study the martensitic transformation in its "pure form", as shown in previous parts of this paper.

Martensitic transformations are perhaps always accompanied by plastic deformation. Plastic deformation is the main mechanism of the dissipation of the elastic energy during martensitic phase transformations. If the quantity of plastic deformation is small, the transformation is reversible and, being well organized, has a small hysteresis. If the quantity of plastic deformation increases, the dissipated energy increases, hysteresis grows and the reversibility of the transformation decreases. Thus the martensitic transformation cannot be understood without careful study of the deformation process. Therefore, experimental and theoretical studies on the transformational plasticity are very important $[32,33]$.

\section{CONCLUSION}

At ICOMAT-77 and in the review papers [1,2], I had attempted to depict the conceptual picture of the martensitic transformation. Now many particular issues have been clarified and some new results have been discovered, especially in the area of the effect of external stress on the transformation. However, my general understanding of the martensitic transformation has not been changed significantly. This paper is the logical conclusion of these attempts.

As a young scientist, I was fascinated with the task which was formulated by my teacher Georgiy Kurdjumov. He had established and experimentally proved the fundamental concepts of the martensitic phenomenon: strain character of the lattice reconstruction and coherency of a heterophase state. The task is to explain all the peculiarities of martensitic transformation on the basis of these fundamental facts. I think that this task has been accomplished now. The theory presented here satisfactorily explains the phenomena observed without the use of additional assumptions or special models. It makes the theory universal and applicable in a greater extent to all phase transformations in solids. However, a great deal is left to be done in the development and improvement of the theory to transform it into quantitative one which can act as dependable tool for engineering of novel materials. I have tried to mention some of the current problems in the paper.

\section{ACKNOWLEDGMENT}

I am grateful to Julia Slutsker, S. Pamir Alpay, and Scott A. Mathews for their kind help in writing this paper.

\section{REFERENCES}

[1] Roitburd, A.L., Solid State Physics 33 (1978) 317.

[2] Roitburd, A.L. and Kurdjumov, G.L., Mat. Sci. Engr. 39 (1979) 141.

[3] Olson, G.B., these proceedings.

[4] Kurdjumov, G.V., Dokl. Akad. Nauk SSSR 60 (1948) 1543.

[5] Roitburd, A.L., Works of Central Res. Inst. of Ferrous Metallurgy 4 (1960) 56.

[6] Roitburd, A.L., Sov. Phys.-Crystallogr. 7 (1962) 226

[7] Roitburd A.L., In Problems of Modern Crystallography, B. Vainstein and A. Chernov Eds., (Nauka, 1975).

[8] Barsch, G.R. and Krumhansl, J.A., in Martensite, G.B. Olson and W.S. Owen Eds. (ASM, 1992) 125.

[9] Barsch, G.R., these proceedings; see also papers by Saxena A. and Hatch D.M..

[10] Umansev, A.R. and Roitburd, A.L., Sov. Phys.-Solid State 30 (1988) 651. 
[11] Olson G.B. and Roytburd A.L., in Martensite, G.B. Olson and W.S. Owen Eds. (ASM, 1992) 149.

[12] Roitburd, A.L., Mat. Sci. Engr. A127 (1990) 229.

[13] Abeyaratne R and Knowles J.K., Archs Ration. Mech. Analysis 114 (1991) 119; Quart. Appl. Math. 50 (1992) 149.

[14] Truskinovsky L., in Shock Induced Transition and Phase Structures, R. Fosdick, E. Dunn and M. Slemrod Eds. 52 (IMA 1993) 185.

[15] Roytburd, A.L., Phase Trans. 45 (1993) 1.

[16] Roitburd A.L. and Yu Y., in Twinning, Yoo M.H. and Wuttig M. Eds. (TMS, 1993) 217.

[17] Roitburd, A.L., Sov. Phys. Uspekhi 17 (1974) 326.

[18] Roytburd A.L. and Slutsker J., J. Appl. Phys. 77 (1995) 27.; Roytburd A.L., "Deformation through Transformation", Proc. MECAMAT 95, J. de Physique Colloque series (Les Editions de Physique, Les Ulis, in press).

[19] Muller I. and Xu H., Acta Metall. 39 (1991) 263.

[20] Bendersky L.A., Roytburd A. L. and Boettinger W. J., Acta Metall 42 (1994) 2323.

[21] Weschler, M.S., Lieberman D.S. and Read T.A., Trans AIME 197 (1953) 1503.

[22] Wayman C.M. , Introduction to Crystallography of Martensitic Transformation (MacMillan, 1964).

[23] Ball J.M. and James R.D., Phil. Trans. R. Soc. 338A (1992) 389; Ball J.M. and James R.D. Proc. ICOMAT-92, Wayman C.M. and Perkins J. Eds (Montery, 1993) 65.

[24] Bhattacharya K., Archs Ration. Mech. Analysis 120 (1992) 201.

[25] Chu C. and James R.D., these proceedings.

[26] Roitburd A.L. and Kosenko N.S., Scripta Met. 11 (1977) 1039.

[27] Roitburd A.L. and Pankova M.N., Phys. Metall. Metall. 59 (1985) 131.

[28] Khachaturyan A.G., Theory of Structural Transformations in Solids (John Wiley and Sons, 1983).

[29] Roytburd A.L., Proc. ICOMAT-92, Wayman C.M. and Perkins J. Eds (Montery, 1993) 37; Rotyburd A.L., Wuttig M. and Zhukovskiy I., Scripta Met. 27 (1992) 1343.

[30] Bhattacharya K. and R.V. Kohn, these proceedings.

[31] Khachaturyan A.G., Shapiro S.M. and Semenovskaya S., Phys. Rev. B 43 (1991) 10832.

[32] Gautier E., these proceedings.

[33] Levitas V., these proceedings. 